\title{
Fibonacci Sequence-related Phenomenon During Chondrocyte Proliferation of the Gottingen Pig Knee Tibial Plateau
}

xiao jian wang ( $\nabla$ wxj990713@163.com )

Shanxi Medical University

Wei Tian

Shanxi Provincial Peoples Hospital

Jian-bo Wu

Shanxi Provincial Peoples Hospital

Jian Zhang

Shanxi Provincial Peoples Hospital

\section{Research Article}

Keywords: Chondrocytes, Cell proliferation, Fibonacci sequence, Gottingen pigs

Posted Date: September 28th, 2021

DOI: https://doi.org/10.21203/rs.3.rs-929643/v1

License: (a) (i) This work is licensed under a Creative Commons Attribution 4.0 International License. Read Full License 


\section{Abstract}

Background The aim of our study was to observe the quantitative changes in tibial plateau chondrocytes in the proliferation process from normal Gottingen pigs in vitro and compare them with the Fibonacci sequence.

Methods Chondrocytes from normal Gottingen pig tibial plateau cartilage were isolated and cultured to the third generation, and the number of chondrocytes was measured to determine whether the chondrocyte growth was at the logarithmic growth phase. Chondrocytes were added to cell culture bottles at very a low density to allow full separation and allow each chondrocyte to grow as independently as possible. Thirty single chondrocytes were selected, and the number of chondrocyte colonies were observed and recorded every day.

Results Among the 30 chondrocyte colonies, the changes in the numbers of 16 chondrocyte colonies were $1,2,3,5,8,13,21,34,55,89,144,233,377,610$, and 987 , which conformed to the numbers of the Fibonacci sequence.The number of other chondrocyte colonies was lower than corresponding numbers of the Fibonacci sequence at the same time point.

Conclusion The numbers of normal Gottingen pig tibial plateau chondrocytes during the proliferation process were in line with the numbers of the Fibonacci sequence.Alignment to the Fibonacci sequence may be the fastest way for changes in the numbers of normal chondrocytes during the proliferation process in vitro.

\section{Background}

The Fibonacci sequence was first described by the Italian mathematician Leonardo Fibonacci ${ }^{1}$. He defined the sequence 0,1 as the first two terms, and the numerical value of the third term is the sum of the first two terms, namely, $a_{1}=0 a_{2}=1, a_{n}=a_{n-2}+a_{n-1}$. The specific values are $0,1,1,2,3,5,8,13,21,34,55$, $89,144 \ldots$ Later, it was found that the Fibonacci sequence is closely related to the biological development of animals and plants in nature ${ }^{2-4}$, such as the number of branches of large trees, the number of leaves of plants, the spiral lines of snail shells and sunflower seeds.Some scholars believe that when an individual has a certain amount of energy, the proliferation mode conforming to the Fibonacci sequence may be fastest.

The proliferation of chondrocytes on the tibial plateau of the knee joint has always been a popular research topic ${ }^{5-7}$. Studies have shown that when knee joint degenerative diseases occur, the proliferation ability of chondrocytes in tibial plateau cartilage is obviously weakened, which eventually leads to cartilage degeneration ${ }^{8-11}$. When we cultured tibial plateau chondrocytes of Gottingen pigs in vitro, we found that the chondrocyte number of the proliferation process was closely related to the Fibonacci sequence. 
The aim of our study was to observe whether the Gottingen pig tibial plateau chondrocyte number of the proliferation processwas in line with the Fibonacci sequence.

\section{Materials And Methods \\ Animal handling}

This study was approved by the Ethics Committee of Shanxi Medical University (approval number:SXMUE2019004). Ten 6-month-old female specific-pathogen free (SPF)-grade Gottingen pigs (Animal Experiment Center of Shanxi Provincial People's Hospital, China) were euthanized, and cartilage tissue samples of the bilateral knee tibial plateau were obtained under aseptic conditions. All experiments were performed in accordance with the Public Health Service Policyand the Guide to the Care and Use of Laboratory Animals.Cartilage tissue of the Gottingen pig osteoarthritis(OA) knee was subsequently obtained (via the anterior cruciate ligament cutting model,provided by Professor Wei Lei of Brown University).

\section{Tibial growth plate chondrocytes}

A sterile scalpel was used to cut layers of cartilage tissue in both the normal group and OA group, and chondrocytes were obtained after tissue clipping and collagenase type II digestion. The isolated chondrocytes were cultured in Dulbecco's modified Eagle's medium (DMEM) supplemented with 10\% foetal calf serum (FCS) at $37^{\circ} \mathrm{C}$ in a humidified atmosphere comprising $95 \%$ air and $5 \% \mathrm{CO}_{2}$. The chondrocytes were cultured to the third generation (P3) for experiments.

\section{Planting and observation of chondrocytes}

The chondrocytes were grown to maturity and added to a T75 culture flask at very low density $\left(10^{4} /\right.$ bottle).A total of 10 bottles of chondrocytes and a total of 30 chondrocyte colonies were used for experiments in the normal group and OA group,respectively.After 24 hours of chondrocyte adhesion, three isolated cells were selected from each bottle for observations and moved to the bottom wall of the culture bottle for subsequent observations. Every day, the changes in the number of all chondrocyte colonies were observed, and the corresponding time points were recorded.

\section{Comparison of chondrocyte proliferation between the normal group and the OA group}

The change in the number of chondrocyte colonies in the normal group and OA group was plotted with observation days and compared with the Fibonacci sequence, and the average norm was calculated as follows:Average norm $=\left[\left(N_{1}-1\right)^{2}+\left(N_{2}-2\right)^{2}+\left(N_{3}-3\right)^{2}+\left(N_{4}-5\right)^{2}+\left(N_{5}-8\right)^{2}+\left(N_{6}-13\right)^{2}+\left(N_{7}-21\right)^{2}+\left(N_{8}-34\right)^{2}+\right.$ $\left.\left(N_{9}-55\right)^{2}+\left(N_{10}-89\right)^{2}+\left(N_{11}-144\right)^{2}+\left(N_{12}-233\right)^{2}+\left(N_{13}-377\right)^{2}+\left(N_{14}-610\right)^{2}+\left(N_{15}-987\right)^{2}\right] / 30$ (N represents the number of chondrocytes at the observed time point). 


\section{Results}

\section{Comparison of normal chondrocyte proliferation with the Fibonacci sequence}

Among the 30 chondrocyte colonies, the changes in the number of 16 chondrocyte colonies were 1, 2, 3, $5,8,13,21,34,55,89,144,233,377,610,987$ at the corresponding time point, which conformed to the Fibonacci sequence.The number of other chondrocyte colonies was lower than that of the Fibonacci sequence at the same time point (Table 1, Fig. 1).

\section{Comparison of normal chondrocyte proliferation with OA chondrocytes}

Compared with that of normal chondrocytes, the average norm of chondrocytes in the OA group increased significantly. This means that the proliferation number of chondrocytes in the OA group is highly different from the Fibonacci sequence. The proliferation number of chondrocytes in the normal group was closer to the Fibonacci sequence. The 24-hour average proliferation rate was lower in the OA group than in the normal group (Table 2, Fig. 1).

\section{Discussion}

The Fibonacci sequence is also called the "rabbit sequence" ${ }^{12}$. The earliest model problem is as follows: Suppose there is a pair of young rabbits that grow up after one month, and then they can breed a pair of young rabbits every month. The young rabbits will also grow up after one month of growth and join the ranks of young breeding rabbits. Assuming that no rabbits will die, ask how many pairs of rabbits there are in month X.According to the calculation, the specific values are 1 pair in the first month, 2 pairs in the second month, 3 pairs in the third month, 5 pairs in the fourth month, 8 pairs in the fifth month, 13 pairs in the sixth month, 21 pairs in the seventh month, 34 pairs in the eighth month and 55 pairs in the ninth month, .... Since then, scholars have found that the processes of several aspects of many species in nature follow the Fibonacci sequence, such as the emergence of large branches, which is as follows: the thickest trunk is 1 , the upward branches are 2 , and then the upward branches are $3,5,8$,... For example, the emergence of some plant leaves and the number of petals are consistent with the numbers of the sequence. Why does this phenomenon occur? Scientists have found through experiments that breeding processes in line with the Fibonacci sequence are the fastest way,as the energy in animals and plants is constant this way 2,313 .

When knee OA occurs, the proliferation ability of chondrocytes in articular cartilage tissue decreases, which leads to a decrease in both chondrocytes and extracellular matrix secreted by chondrocytes,ultimately leading to a decrease in cartilage tissue quantity.Therefore, the proliferation ability of chondrocytes is an important direction to study the development of knee $O A^{14,15}$.The division and proliferation of chondrocytes are affected by many factors. When we cultured chondrocytes in vitro, 
we found that the process of chondrocyte division and proliferation did not follow the mode of 1-2,24,4-8; in other words, it was not a $2^{n}$ series mode.Instead, we found that when a chondrocyte population divides and proliferates, there are always some chondrocytes in the division stage and the other chondrocytes in the resting stage.As shown by the red arrow in Fig. 1F, 3 out of 5 chondrocytes are in the cell division stage (the nucleus is about to divide).

To observe the change in the proliferation number of normal chondrocytes,we added Gottingen pig knee chondrocytes to culture bottles at very low density,and because of the "ameba" movement of chondrocytes, when we selected chondrocytes for observation, we selected isolated chondrocytes without other cells around the microscope field of vision.After 42 days of observation, we found that the proliferation of normal chondrocytes conformed to the Fibonacci sequence. Sixteen of the 30 observed chondrocyte colonies essentially conformed to the sequence. The proliferation number of the other remaining chondrocytes was lower than that of the Fibonacci sequence, and no chondrocyte colony presenting numbers exceeding those corresponding to this sequence was observed.Moreover, the chondrocytes in the OA group essentially did not have chondrocyte colonies whose growth followed a pattern similar to that of the Fibonacci sequence, and the number of chondrocyte colonies that proliferated in the end was significantly less than that in the normal group.By comparing the average norm of chondrocyte number and Fibonacci number at the same time point between the two groups, we found that the average norm of the OA group was significantly larger than that of the normal group.This shows that the proliferation number of chondrocytes in the OA group deviated from thenumbers of the Fibonacci sequence more than that in the normal group did.Therefore, we believe that in the process of the proliferation of Gottingen pig knee chondrocytes, the change in chondrocyte number is close to the numbers in the Fibonacci sequence under normal circumstances, and when there are interferingfactors such as cell ageing and the proliferation ability decreases, the number deviates from and becomes lower than corresponding numbers of the Fibonacci sequence.We even speculate that the fastest of all cell proliferation methods may be closely aligned to the Fibonacci sequence, but the time differences in changes in cell numbers are different, which eventually leads to the inconsistency of the cell proliferation rate after 24 hours.

\section{Conclusion}

In summary,we found that the changes in the number of Gottingen pig knee normal chondrocytes in vitro were very consistent with the Fibonacci sequence, while OA chondrocytes deviated from and were lower than the corresponding numbers of this sequence. The cell proliferation mode conforming to the Fibonacci sequence may be the fastest cell proliferation mode.

Our experiment also has some limitations. In this experiment, we found that the change in the number of chondrocytes on the tibial plateau of normal knees in vitro is in accordance with the numbers of the Fibonacci sequence. However,the longest observation time was 42 days, and the apoptosis rate of chondrocytes was not considered. What is the additional relationship between chondrocyte proliferation and the Fibonacci sequence? Further observations and research are needed. 


\section{Abbreviations}

OA: Osteoarthritis; OARSI: Osteoarthritis Research Society International (OARSI); NS: normal saline; SPF: Specific-pathogen free; DMEM: Dulbecco's modified Eagle's medium; FCS: Fetal calf serum; P3: Third generation

\section{Declarations}

\section{Acknowledgements}

The authors would like to thank Fang Liu for her work on literature search and query. The author also thanks Gang Zheng and Hong Bi for their work on pathology.

\section{Funding source}

This work was supported by grants from the China Postdoctoral Science Foundation (2020M680910), Fund Program for the Scientific Activities of Selected Returned Overseas Professionals in Shanxi Province (no.20210019).

\section{Availability of data and materials}

Not applicable

\section{Authors' contributions}

XJ Wang: study design, data acquisition, data analysis, wrote the manuscript. Wei Tian: statistical analyses.JB Wu and J Zhang: study design, manuscript correction. All authors have approved the submitted manuscript.

\section{Competing interests}

None declared.

\section{Consent for publication}

Not applicable. 


\section{Ethical Approval and Consent to participat}

This study was approved by the Ethics Committee of Shanxi Medical University [Approval number: SXMUE(2019004)] (Tai Yuan,China).

\section{References}

1. Luttge U, Souza GM. The Golden Section and beauty in nature: The perfection of symmetry and the charm of asymmetry. Prog Biophys Mol Biol. 2019;146:98-103.

2. Lachowiec J, Queitsch C, Kliebenstein DJ. Molecular mechanisms governing differential robustness of development and environmental responses in plants. Ann Bot. 2016;117:795-809.

3. Gliroy S, Trewavas A. Signal processing and transduction in plant cells: the end of the beginning? Nat Rev Mol Cell Biol. 2001;2:307-14.

4. Kirisci M, Karaisa A. Fibonacci statistical convergence and Korovkin type approximation theorems. J Inequal Appl. 2017;2017:229.

5. Abram SGF. Judge A,Beard DJ,et al.Rates of knee arthroplasty within one-year of undergoing arthroscopic partial meniscectomy in England: temporal trends, regional and age-group variation in conversion rates. Osteoarthritis Cartilage. 2019;27:1420-9.

6. Murray IR, Benke MT, Mandelbaum BR. Management of knee articular cartilage injuries in athletes: chondroprotection, chondrofacilitation, and resurfacing. Knee Surg Sports Traumatol Arthrosc. 2016;24:1617-26.

7. Guermazi A, Roemer FW, Burstein D, et al. Why radiography should no longer be considered a surrogate outcome measure for longitudinal assessment of cartilage in knee osteoarthritis. Arthritis Res Ther. 2011;13:247.

8. Stiffel V, Rundle $\mathrm{CH}$, Sheng $\mathrm{MH}$, et al. A Mouse Noninvasive Intraarticular Tibial Plateau Compression Loading-Induced Injury Model of Posttraumatic Osteoarthritis. Calcif Tissue In. 2020;106:158-71.

9. Hee J, Chaekyu K, Remi-Martin L, et al. Local Clearance of Senescent Cells Attenuates the Development of Post-Traumatic Osteoarthritis and Creates a Pro-Regenerative Environment. Nat Med. 2017;23:775-81.

10. Didier P, David G, Daniela P,et al. p16INK4a and Its Regulator miR-24 Link Senescence and Chondrocyte Terminal Differentiation-Associated Matrix Remodeling in Osteoarthritis. Arthritis Res Ther. 2014;16:R58.

11. Xue JF, Shi ZM, Zou J, et al. Inhibition of PI3K/AKT/mTOR signaling pathway promotes autophagy of articular chondrocytes and attenuates inflammatory response in rats with osteoarthritis. Biomed Pharmacother. 2017;89:1252-61.

12. Kirişci M, Karaisa A. Fibonacci statistical convergence and Korovkin type approximation theorems. Inequal Appl. 2017;2017:229. 
13. Lugito G, Nagarajan S, Woo EM. Explosive Fibonacci-sequence growth into unusual sector-face morphology in poly(L-lactic acid) crystallized with polymeric diluents. Sci Rep. 2020;10:10811.

14. Carballo CB, Nakagawa Y, Sekiya I, et al. Basic Science of Articular Cartilage. Clin Sports Med. 2017;36:413-25.

15. Liu Q, Li M, Jiang L, et al.METTL3 promotes experimental osteoarthritis development by regulating inflammatory response and apoptosis in chondrocyte. Biochem. Biophys Res Commun. 2019;516:22-7.

\section{Tables}

Due to technical limitations, tables are only available as a download in the Supplemental Files section.

\section{Figures}

A

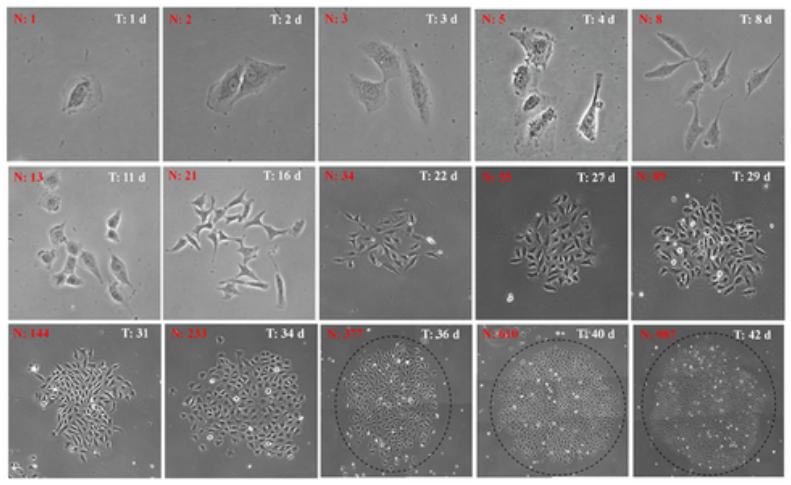

B

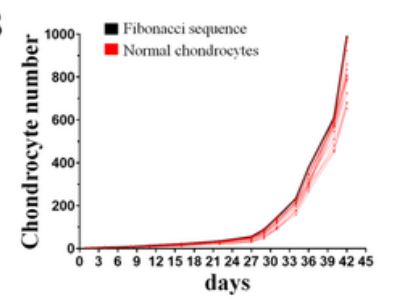

C
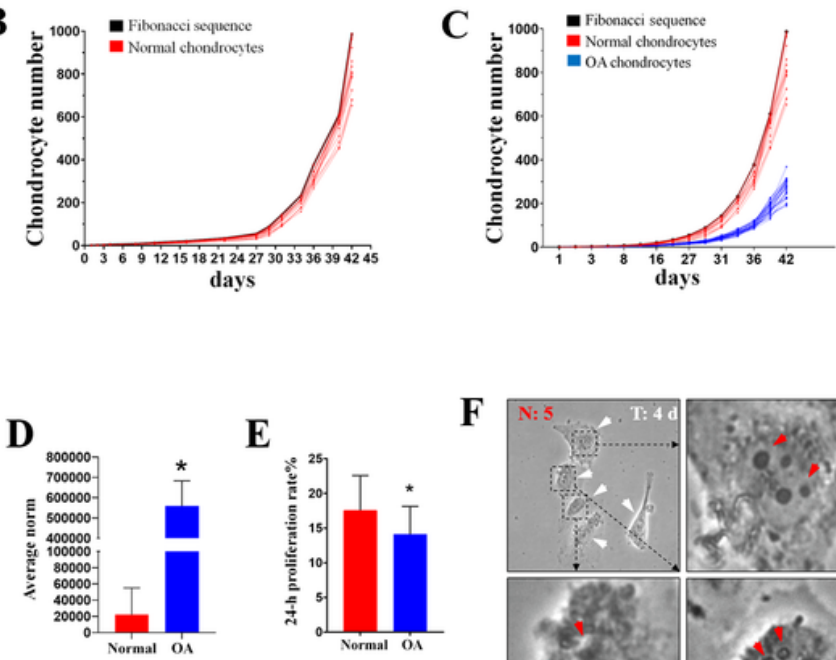

$\mathbf{F}$

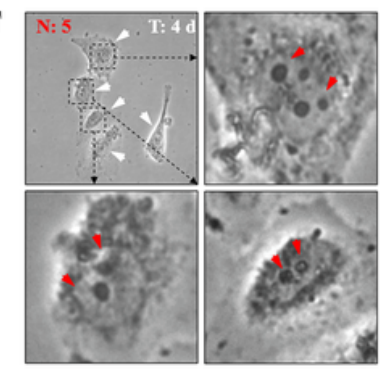

Figure1

\section{Figure 1}

Gottingen pig knee tibial plateau chondrocytes cultured in vitro and the Fibonacci sequence. (A) Changes in chondrocyte number and morphology at 42 days. (B)Comparison between the proliferation of normal chondrocytes and the Fibonacci sequence.(C)The proliferation curves of normal chondrocytes and OA chondrocytes were compared with the Fibonacci sequence.(D)Comparison of the average norm with the Fibonacci sequence in both the normal chondrocyte group and the OA chondrocyte group.(E)Comparison of the 24-h proliferation rate between normal chondrocytes and OA chondrocytes.(F)Three(red arrows) of the five chondrocytes(white arrows) are in a state of division. 


\section{Supplementary Files}

This is a list of supplementary files associated with this preprint. Click to download.

- Table1.jpg

- Table2.jpg 